\title{
Advanced Homology Computation of Digital Volumes Via Cell Complexes ${ }^{\star}$
}

\author{
Helena Molina-Abril and Pedro Real \\ Dpto. Matematica Aplicada I, E.T.S.I. Informatica, Universidad de Sevilla, \\ Avda. Reina Mercedes, s/n 41012 Sevilla (Spain) \\ $\{$ habril,real\}@us.es
}

\begin{abstract}
Given a 3D binary voxel-based digital object $V$, an algorithm for computing homological information for $V$ via a polyhedral cell complex is designed. By homological information we understand not only Betti numbers, representative cycles of homology classes and homological classification of cycles but also the computation of homology numbers related additional algebraic structures defined on homology (coproduct in homology, product in cohomology, (co)homology operations,...). The algorithm is mainly based on the following facts: a) a local 3 D-polyhedrization of any $2 \times 2 \times 2$ configuration of mutually 26 -adjacent black voxels providing a coherent cell complex at global level; b) a description of the homology of a digital volume as an algebraic-gradient vector field on the cell complex (see Discrete Morse Theory [5,AT-model method [7/5]). Saving this vector field, we go further obtaining homological information at no extra time processing cost.
\end{abstract}

\section{Introduction}

One possible way for developing techniques for volume recognition can be through relevant structural descriptors, mainly based on topological-geometrical properties. Topology deals with connectivity and separability features and although the global nature of topological properties makes their computation difficult, topology seems to be an essential part of the vocabulary by which human visual system represent and characterize objects [2]. Homology gives us the simplest topological version of a volume in terms of information about connected components, tunnels or holes and cavities on it. Homological numbers commonly used in Pattern Recognition as robust descriptors are Betti numbers (number of connected components, holes and cavities), local topological characterization of voxels and Euler characteristic. Nowadays, the current interactions between Pattern Recognition and Computational Topology are mainly at graph-based level and it includes issues such that topological skeletonization and Reeb graphs [1]. Examples of image analysis and pattern recognition problems that benefit from the use of topological considerations include, for example, the use of homology invariants to compare

\footnotetext{
* This work has been partially supported by "Computational Topology and Applied Mathematics" PAICYT research project FQM-296, "Andalusian research project PO6-TIC-02268) and Spanish MEC project MTM2006-03722.
} 
objects, for image classification, or shape recognition. At present, there is an intensive research on homology computation in the context of the image (9) for cubical homology, 15,16 for persistent homology, 3. for combinatorial maps, 12 for matrix methods, [13] for graph pyramids, [7] for algebraic-topological models).Even so, the amount of efficient numerical tools combining homological with geometrical information could be increased using and saving additional algebraic data structures at no extra computational time.

In this paper, we establish a method for computing homology numbers for a binary 26-digital volume $V$. 3D digital homology is developed here in a cell (more precisely, polyhedral) ambiance. Roughly speaking, a continuous analogous $K(V)$ of $V$ is a cell complex having the same "homology" than it. In particular, the homology of $V$ and $K(V)$ are isomorphics and all the numerical descriptors derived from homological and geometrical information in both objects are equals (Betti numbers, Euler characteristic,...) or extremely similar (size and orientation of geometrically well-adapted representative cycles of homology generators,...). Due to the fact that we encode homology in terms of an algebraic gradient vector field on the cell complex, it is possible to deduce not only Betti numbers, representative cycles of homology classes and homological classification of cycles but also homology numbers related to additional algebraic structures defined on homology (coproduct in homology, product in cohomology, (co)homology operations,...). In discrete context, this idea of encapsulating "homology" under an algebraic-topological format has already been used in theories like AT-model [6] and Discrete Morse theory [5]. For calculating the homology gradient on $K(V)$, it is possible to adequately modify classical algorithms (Smith Normal Form, incremental, etc) for computing homology. We deal with here the incremental Delfinado-Edelsbrunner technique.

The remainder of this paper is organised as follows. In section 2 we construct the cell complex $K(V)$. Definition and computation of the homology gradient vector field is given in section 3. Then we briefly comment some advantages of saving this algebraic information in section 4 . We focus our interest in the canonical coproduct in homology [11].

\section{3D Polyhedrization}

In algebraic topology, specifically homotopy theory, shapes are often constructed out of contractile cells (having the homotopy type of a point) of different dimension which are coherently attached one to each other. Intuitively, a contractile cell is one that can be continuously shrunk to a point. In that way, cell complexes appear (see [1] for a formal definition) and constitute a class of spaces that is broader than simplicial complexes. They retain a combinatorial nature, so that computational consideration can still be applied in that context. In fact, the cell complex generalizes both the notion of graph adding cells of dimension greater than 1 as well as the notion of a simplicial complex in that triangles become polygons and tetrahedra become polyhedra. Note also that the cells need not be straight, such that a polygon may contain as few as a single edge connecting a 

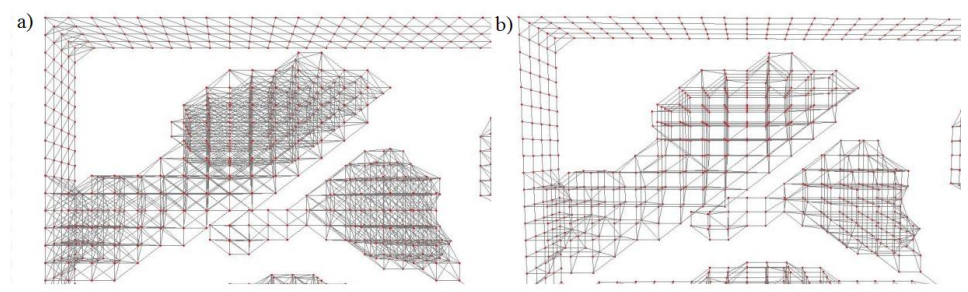

Fig. 1. A simplicial complex (a) and a cell complex (b)

single vertex to itself, and a polyhedron may contain as few as a single face, a single vertex and no edges.

A cell complex or simply complex in $\mathbf{R}^{d}$ is a set $K=\left\{K_{q}\right\}_{q \geq 0}$ of convex polyhedra (called cells). A face of a cell consist of each lower dimension cell, which make it up, in satisfying two conditions: (1) Every face of a cell is a cell (i.e. in $K$ ), and (2) If $\sigma$ and $\sigma^{\prime}$ are cells, then their intersection is a common face of both. A simplicial complex is a cell complex whose cells are all simplices.

We associate here a three-dimensional cell complex $K(V)$ to a digital object $V$ with 26-adjacency relationship. The complex $K(V)$ and the cubical complex canonically associated to $V$ consisting in the set of geometric realizations of the voxels forming $V$ are homotopically equivalent, and consequently, present the same homological information. To obtain the cell complex $K(V)$ we do as follows. Each black voxel can be seen as a point (0-cell) of our complex. The algorithm consist on dividing the volume into overlapped (its intersection being a "square" of four voxels mutually 26-adjacent) unit cubes formed by eight voxels mutually 26-adjacents, and to associate each unit cube configuration with its corresponding cell. We scan the complete volume, always taking as elementary step a unit cube.

The cell associated to a unit cube configuration is a 0 -cell if there is a single point. If there are two points, the complex is a 1-cell which is the edge conecting both of them. With three or four coplanar points on the set, the 2-cell associated is a polygon. If there are four non coplanar points or more, the 3 -cell is a polyhedra. In other words, the cell associated to a unit cube configuration is just

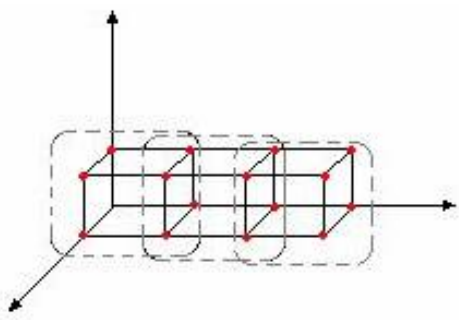

Fig. 2. 
the convex hull of the black points and all its lower dimension faces. Note that for 3-cells, their 2-dimension faces are either triangles or squares.

Once we have covered all the volume and joined all the cells, we build the complete cell complex without incoherences.

Our method gives us the possibility of canonically associate a simplicial complex equivalent to $V$ and this fact allows us to define in a straightforward manner the boundary operator of each cell in the complex. For doing that, we limit ourselves to say that we here slightly modify the idea proposed in [10] to create a new triangulation method on the surface of a digital volume. Because a $2 \times 2 \times 2$ volume includes eight points, each of which can be black or white, there are 256 possible patterns in a unit cube. If we ignore the congruent patterns differing only by the rotations of the center of the unit cube, the 256 patterns can be reduced to 23 .

On a simplicial complex, a 0 -simplex (v0) is a point; a 1-simplex (v0, v1) is a line connecting vertex v0 and v1; a 2-simplex (v0, v1, v2) is the triangle defined by these three vertex; and a 3-simplex (v0, v1, v2, v3) is a tetrahedra. The aim is to divide each cell into points, edges, triangles or tetraedra to build the simplicial complex.

Using 26-adjacency to divide each cell, we will find crossing simplex when there are four coplanar points into the cube. In all these cases we will choose one direction we will give preference, to avoid simplex crossing.

The problematic situations arise only when there are four or more black points into a $2 \times 2 \times 2$ set, so we have to study each possibility separately.

As is shown in figure 3 , given a cube with five black points, there are three possible positions of this points not considering cube rotations.

In figure 3.a we have to choose a direction, such that no incoherencies will appear between one cube and their neighbours. That is why all possible rotations of this point configuration must be considered separately, because depending on the cube's face we find the four coplanar points, we should choose a different tetrahedrization, according to the decision adopted for the four points. We have then six different configurations to consider for this case.

The other two situations (3.b, 3.c) are easier to carry out, because we can apply the tetrahedrization showed in the picture, to all of the possible rotations in each case without incoherencies.

With six black points, there are also three possible configurations.In two of them we have to consider every rotation separately, and there are twelve possibilities for each one. When there are seven points, we have just eight possibilities, and we should build a different tetrahedralization for each one. Finally, when all the points are black into the cube, there is only one possible tetrahedrization

a)

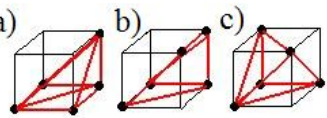

Fig. 3. Five black points 


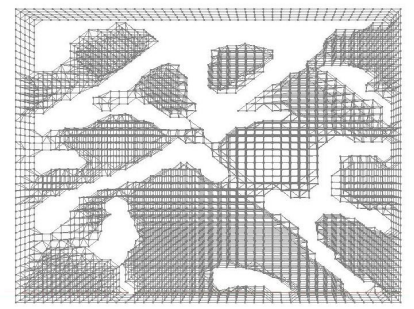

Fig. 4. Cell complex $K(V)$ of a trabecular bone volume with 21938 cells

according to the direction we chose for each cube's face. Once we have the entire cube's volume and their simplicial complex, we have to build a general simplicial complex for the total volume.

If we just join the different complexes, taking into account not to take two times the same simplex, we will not find incoherences obtaining the simplicial final complex.

\section{Homology Gradient Vector Field for a 3D Digital Object}

In this section we extend the Discrete Morse Theory notion of gradient vector field of a Morse function [5. Homology is encoded here in terms of an algebraic vector field (so-called homology gradient vector field) from which it is possible to compute not only Betti numbers (number of connected components, holes and cavities), representative cycles of homology classes and homological classification of cycles but also homology numbers related to additional algebraic structures defined on homology (coproduct in homology, product in cohomology, (co)homology operations,...). In discrete setting, this idea of considering a linear map for defining homology has been extensively developed in Discrete Morse and AT-model [7] theories. In the first one, this map is a vector field and in the second one, it is a chain homotopy operator determining a strong algebraic relationship between the 3D object and its homology. Here, we suitably combine concepts and techniques of both theories in order to specify an adequate framework for efficiently computing homological information.

To explain the notion of homology gradient vector field, we first describe some elementary concepts from Homological Algebra (see [8]).

Since the objects considered in this paper are embedded in $\mathbf{R}^{3}$ then the homology groups vanish for dimensions greater than 3 and they are torsion-free for dimensions 0,1 and 2. Throughout the paper, we consider that the ground ring is a finite field $\mathbf{F}$. Let $K$ be a three-dimensional cell complex. A $q$-chain a is a formal sum of simplices of $K^{(q)}$. We denote $\sigma \in a$ if $\sigma \in K^{(q)}$ is a summand of $a$. The $q$-chains form a group with respect to the component-wise addition; this group is the $q$ th chain group of $K$, denoted by $C_{q}(K)$. There is a chain group for every integer $q \geq 0$, but for a complex in $\mathbf{R}^{3}$, only the ones for $0 \leq q \leq 3$ may be non-trivial. 
The boundary map $\partial_{q}: C_{q}(K) \rightarrow C_{q-1}(K)$ applied to a $q$-cell $\sigma$ gives us the collection of all its $(q-1)$-faces which is a $(q-1)$-chain. By linearity, the boundary operator $\partial_{q}$ can be extended to $q$-chains. In the concrete case of a simplicial complex, the boundary of a $q$-simplex defined in terms of vertices $\sigma=\left\langle v_{0}, \ldots, v_{q}\right\rangle$ is defined by: $\partial_{q}(\sigma)=\sum\left\langle v_{0}, \ldots, \hat{v}_{i}, \ldots, v_{q}\right\rangle$, where the hat means that vertex $v_{i}$ is omitted. In our case, taking into account that the 3 -cells of our cell complexes can automatically be subdivided into tetrahedra, its boundary map can directly be derived from that of the component tetrahedra. It is clear that $\partial_{q-1} \partial_{q}=0$. A chain $a \in C_{q}$ is called a $q-$ cycle if $\partial_{-} q(a)=0$. If $a=\partial_{-} q+1\left(a^{\prime}\right)$ for some $a^{\prime} \in C_{q+1}$ then $a$ is called a $q$-boundary. Define the $q$ th homology group to be the quotient group of $q$-cycles and $q$-boundaries, denoted by $H_{q}(\mathcal{C})$. Let $\mathcal{C}=\left\{C_{q}, \partial_{q}\right\}$ and $\mathcal{C}^{\prime}=\left\{C_{q}^{\prime}, \partial_{q}^{\prime}\right\}$ be two chain complexes. A chain map $f: \mathcal{C} \rightarrow \mathcal{C}^{\prime}$ is a family of homomorphisms $\left\{f_{q}: C_{q} \rightarrow C_{q}^{\prime}\right\}_{q \geq 0}$ such that $\partial_{q}^{\prime} f_{q}=f_{q-1} \partial_{q}$.

Now, we are ready for giving an algebraic version of the Discrete Morse Theory notion of gradient vector field, which is not necessarily associated with a Morse function. ([5]).

Definition 1. [5] Let $K$ be a finite cell complex. A linear map of chains $\phi$ : $C_{*}(K) \rightarrow C_{*+1}(K)$ is a gradient vector field on $K$ if the following conditions hold:

1. For any cell $a \in K_{q}, \phi(a)$ is a $q+1$-cell.

2. $\phi^{2}=0$

The first condition means that $\phi$ is a combinatorial function and the second that $V$ induces the following partition on the $q$-cells of $K$ :

$$
\begin{gathered}
\{q-\text { cells } a \text { of } K\}=\{a \text { such that } \phi(a) \neq 0\} \\
\bigcup\{a \text { such that } a \in \operatorname{Im} \phi\} \bigcup\{a \text { such that } \phi(a)=0 \text { and } a \notin \operatorname{Im} \phi\}
\end{gathered}
$$

Let us note that 0 is an specimen of $C_{*}(K)$. The cells constituting the last set are called critical cells.

In [14, elementary algebraic operations are used for designing an algorithm for computing homology which can be seen as a kind of algebraic-topological thinning. These elementary operations are called integral operators and they can be defined as algebraic versions of the notion gradient vector field (in the sense that $\phi$ applied on a cell gives us not necessarily a cell of higher dimension but possibly a sum of cells).

a)

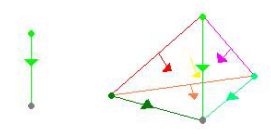

b)

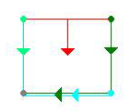

Fig. 5. Combinatorial gradients (a) and algebraic gradients (b) 
The notion of homology gradient vector field of $K$ generalizes that of gradient vector field and integral operator and it condenses all the significant homological information of the cell complex.

Definition 2. A homology (or optimal) gradient vector field for a filtered cell complex $K$ is a linear map of chains $\phi: C_{*}(K) \rightarrow C_{*+1}(K)$ such that $\phi \phi=0$, $\phi \partial \phi=\phi$ and $\partial \phi \partial=\partial$, where $\partial$ is the boundary operator in $C(K)$.

In fact, using homological algebra arguments, it is possible to deduce that $\phi$ determines a strong algebraic relationship connecting $C(K)$ and its homology vector space $H(K)$.

Let $\pi=i d_{\mathcal{C}(\mathcal{K})}-\partial \phi-\phi \partial$. This chain map satisfies that $\pi^{2}=\pi$. If $\operatorname{Im} \pi=\{x \in$ $C(K)$, such that $x=\phi(y)$ for some $y\}$ and $\operatorname{Ker} \pi=\{x \in C(K)$ such that $\phi(x)=$ $0\}$, then $\mathcal{C}(\mathcal{K})=\operatorname{Im} \pi \oplus \operatorname{Ker} \pi)$. Let $f: C(K) \rightarrow \operatorname{Im}(\pi)$ be the corestriction of $\pi$ to $\operatorname{Im}(\pi)$ (that is, $\pi: C(K) \rightarrow \operatorname{Im}(\pi)$ ) and $g: \operatorname{Im}(\pi) \rightarrow C(K)$ be the inclusion. Let $\tilde{d}$ be the boundary operator of $\operatorname{Im}(\pi)$. We now prove that $\tilde{d}=0$. Taking into account that $i d_{C(K)}+g f=\phi d+d \phi, \partial \partial=0$ and $\partial \phi \partial=\partial$, we then obtain $\partial-\partial g f=\partial$. Therefore, $\partial g f=g \tilde{d} f=0$. Since $f$ is onto and $g$ is one- to-one, we deduce that $\tilde{d}=0$. That means that the Morse complex $M_{\phi}=\operatorname{Im} \phi$ is a graded vector space with null boundary operator isomorphic to the homology $H(K)$.

We obtain a homology gradient vector field for $K$ adequately modifying classical algorithms for computing homology (boundary matrix reduction based on Smith Normal Form [1], incremental technique [4],...) existing in the literature. We describe here a homology gradient version of the incremental homology method of Delfinado-Edelsbrunner [4]. For designing such an algorithm it is necessary to impose an order on the set of cells of $K$. We call such an ordering a filter. In other words, given a cell complex $K, \mathcal{K}=\left\{c_{1}, \ldots, c_{m}\right\}$ is a filter if $c_{i}$ is a face of $c_{j}$ for $i<j$. It is possible to "filter" $K$ by first considering all the 0 -cells in a certain order, then an order on all the 1-cells, and so on.

Algorithm 1. Computing a homology gradient vector field for a finite filtered cell complex $K$ (with filter) over a field.

InPUT: The filter $\mathcal{K}_{m}=\left\{c_{0}, \ldots, c_{m}\right\}$ considered on the chain complex $(C(K), \partial)$, and the differential $\partial_{i}$ for the basis $\mathcal{K}_{i}, i=0 \ldots m$.

$\mathcal{C}_{0}:=\left(\left\{c_{0}\right\}, \partial_{0}\right), \quad \phi_{0}\left(c_{0}\right):=0$.

For $i=1$ to $m$ do

$\mathcal{C}_{i}:=\left(\mathcal{C}_{i-1} \cup\left\{c_{i}\right\}, \partial_{i}\right)$,

If $\left(\partial_{i}-\partial_{i-1} \phi_{i-1} \partial_{i}\right)\left(c_{i}\right)=0$, then

$\phi_{i}\left(c_{i}\right):=0$.

For $j=0$ to $i-1$ do, $\phi_{i}\left(c_{j}\right):=\phi_{i-1}\left(c_{j}\right)$.

If $\left(\partial_{i}-\partial_{i-1} \phi_{i-1} \partial_{i}\right)\left(c_{i}\right)$ is a sum of a kind $\sum_{i=1}^{r} \lambda_{i} u_{i} \neq 0 \quad\left(u_{i} \in \mathcal{C}_{i-1}\right)$, then: $\tilde{\phi}\left(u_{1}\right):=-\lambda_{1}^{-1} c_{i}$ and zero in the rest of elements of $\mathcal{C}_{i-1}$, .

then For $j=0$ to $i$ do,

$$
\phi_{i}\left(c_{j}\right)=\left(\phi_{i-1}+\tilde{\phi}-\tilde{\phi} \phi_{i-1} \partial_{i-1}-\tilde{\phi} \partial_{i-1} \phi_{i-1}\right)\left(c_{j}\right),
$$

OUTPUT: a homology gradient vector field $\phi_{m}$ for $K$. 
The algorithm runs in time at most $\mathcal{O}\left(m^{2}\right)$, where $m$ is the number of cells of $K$.

Concerning to the homology computation of a binary digital volume $V$ with 26-adjacency relation, the homology gradient vector field for the canonical 26adjacency cell representation $K(V)$ (with a given filter) of $V$ provides us the desired solution.

This method has already been implemented and has been tested on digital volumes of trabecular bones (courtesy of the Institute of Biomechanics, Zurich, Swiss). The following table shows the Betti numbers and the computation time for the homology of digital trabecular bone volumes of different sizes.

\begin{tabular}{ccc}
\hline Number of cells & Betti Numbers & Time \\
\hline 21938 & $b_{0}=3, b_{1}=12, b_{2}=0$ & $0,885 \mathrm{~s}$. \\
47448 & $b_{0}=2, b_{1}=13, b_{2}=0$ & $2,055 \mathrm{~s}$. \\
117780 & $b_{0}=2, b_{1}=406, b_{2}=13$ & $5,31 \mathrm{s.}$ \\
\hline
\end{tabular}

\section{Advanced Homological Information}

In this section, we briefly comment some advantages of saving a homology gradient vector field $\phi$ for a binary digital volume $V$.

First, considering homology in terms of a linear map $\phi$ increasing the dimension by one allows to give a positive answer to problems so important in object recognition as detecting null-homological cycles (that is, cycles which become zero in homology), homologically classifying cycles at any dimension, to deform a cycle guaranteeing a homological control in the process, reversible topological skeletonization,... In fact, all these question can be handled using as main tool the triple of maps $(\pi, i n c, \phi)$ derived from $\phi$ previously described in the last section. These maps connect in an algebraic strong way the chain complex $C(K(V))$ canonically associated to the polyhedral complex $K(V)$ to its homology $H(V)$. For instance, if we are interested in homologically classify a 1-cycle $c(d(c)=0)$, we only need to compute $\pi(c)$ for determining the homology class it belongs. If $\pi(c)=0$, then $c$ is a boundary and the following equality hold $c=d(\phi(c))$. If we are interested in comparing two different 1-cycles $c$ and $c^{\prime}$ belonging to the same homology class $\pi(c)=\pi\left(c^{\prime}\right)$, we can obtain that $c-c^{\prime}=d \phi\left(c-c^{\prime}\right)$. This means in particular that the 2-chain $\phi\left(c-c^{\prime}\right)$ is a "surface" in $V$ which has as part of its boundary $c$ and $c^{\prime}$. Given a homology class $\pi(c)$, if we are interested in obtaining a specific cycle $c^{\prime}$ belonging to this homology class presenting some properties of geometrical nature (for example, to be "well-adapted" to the geometry of the object), we only need to develop the specific geometric algorithm for getting $c^{\prime}$ and finally to check that $\pi\left(c^{\prime}\right)=\pi(c)$. It is possible to generate a (curve, curve-surface, ....) topological skeleton $S k(K(V))$ which is reversible in the sense that we can reconstitute the cell complex $K(V)$ and consequently, the volume $V$ from the skeleton. In fact, it is possible to design an algorithm f or computing a chain contraction from $C(K(V))$ to $C(S k(K(V))$ which allows us to ensure the reconstruction of a subvolume of $V$ starting from any part of the skeleton. 


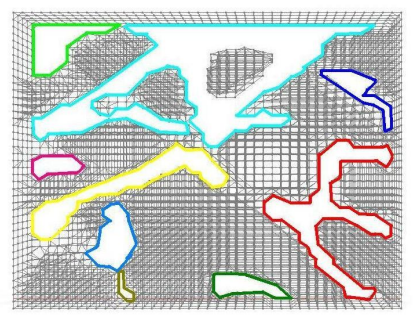

Fig. 6. Geometrically well-adapted 1-cycles

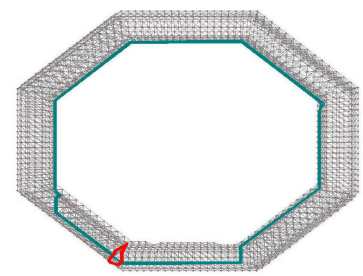

Fig. 7. The hollow torus and the representative cycles of homology classes of dimension 1 and 2

Now, we focus our interest in the computation of the coproduct in homology for simplicially subdivided cell complexes $(K(V)$ is an example of this). There is a canonical coproduct $\Delta: H(V) \rightarrow H(V) \otimes H(V)$ (also called Alexander-Whitney coproduct) in homology which is well defined in the context of Simplicial Topology. This coproduct operation at homology level can be seen as a proper decomposition of 2-cycles into 1-cycles factors. For example in the case of a hollow ring (torus, see the figure), the homology coproduct applied on the global cavity gives us the two fundamental 1-cycles. The formula for this operation at complex chain level applied to a generic triangle $\left(v_{0}, v_{1}, v_{2}\right)$ is the following:

$$
A W\left(v_{0}, v_{1}, v_{2}\right)=\left(v_{0}, v_{1}, v_{2}\right) \otimes\left(v_{2}\right)+\left(v_{0}\right) \otimes\left(v_{0}, v_{1}, v_{2}\right)+\left(v_{0}, v_{1}\right) \otimes\left(v_{1}, v_{2}\right)
$$

This map can be translated to homology level using the triple $(\pi$, inc, $\phi)$. Let $\Delta: H(V) \rightarrow H(V) \otimes H(V)$ be $\Delta([x])=(\pi, \pi) A W(x \times x))$ where $[x]$ means the homology class with representative 2-cycle $x$.

In our case, a cell 2-cycle $c$ we must handle is a sum of cubes and polyhedra, and they can be automatically putted under the form $s$ of sum of the component tetrahedra of them. We use this simplicial description $s$ for the 2-cell cycle in the previous formula for the coproduct.

\section{Conclusions and Future Work}

This paper proposes a polyhedral-based solution to advanced homological computation, opening a door to the design of efficient algorithmic tools which are 
suitable for volume recognition. The fundamental tool in our approach is that of homology gradient vector field which condenses all the relevant homological information of the digital object $V$. This notion is interpreted in a Homological Algebra setting as a chain homotopy equivalence. This strong algebraic "connection" between $V$ and its homology allows to go further in homological computation.

The main theorem of Discrete Morse Theory establish that a cell complex $K$ with discrete Morse function is simple homotopy equivalent to another cellcomplex with exactly one cell of dimension $p$ for each critical cell of dimension $p$. Then, the fundamental idea here is to reduce the number of cells in a complex without changing the homotopy type. In this paper, we construct directly from the boundary operator the homology gradient vector field in an incremental way. If, for each digital object $V$ we want to analyze, it would be possible to coherently install an initial (non-optimal) gradient vector field $\phi_{0}$ in the cubic grid $(2 \times 2 \times 2$ unit cubes) "containing" all the cells of $K(V)$, then we reduce the complexity of the homology calculus, by computing the homology on the Morse complex $M_{\phi_{0}}=\operatorname{Im} \phi_{0}$, exclusively composed of critical cells. The computational costs of the method can drastically be reduced if the number of critical cells of the corresponding Morse complex is small.

\section{References}

1. Biassoti, D., Giorgi, M., Falcidieno, B.: Reeb Graphs for Shape Analysis and Applications. Theoretical Computer Science 392(1-3), 5-22 (2008)

2. Chen, L.: Topological structure in the perception of apparent motion. Perception 14(2), 197-208 (1985)

3. Damiand, G., Peltier, S., Fuchs, L., Lienhardt, P.: Topological Map: An Efficient Tool to Compute Incrementally Topological Features on 3D Images. In: Reulke, R., Eckardt, U., Flach, B., Knauer, U., Polthier, K. (eds.) IWCIA 2006. LNCS, vol. 4040, pp. 1-15. Springer, Heidelberg (2006)

4. Delfinado, C.J.A., Edelsbrunner, H.: An Incremental Algorithm for Betti Numbers of Simplicial Complexes on the 3-Sphere. Comput. Aided Geom. Design 12, 771784 (1995)

5. Forman, R.: A Discrete Morse Theory for Cell Complexes. In: Yau, S.T. (ed.) Geometry, Topology \& Physics for Raoul Bott. International Press (1995)

6. Gonzalez-Diaz, R., Medrano, B., Real, P., Sanchez-Pelaez, J.: Algebraic Topological Analysis pf Time-sequence of Digital Images. In: Ganzha, V.G., Mayr, E.W., Vorozhtsov, E.V. (eds.) CASC 2005. LNCS, vol. 3718, pp. 208-219. Springer, Heidelberg (2005)

7. Gonzalez-Diaz, R., Real, P.: On the Cohomology of $3 D$ Digital Images. Discrete Applied Math. 147, 245-263 (2005)

8. Hatcher, A.: Algebraic Topology. Cambridge University Press, Cambridge (2001)

9. Kaczynski, T., Mischaikow, K., Mrozek, M.: Computational Homology. Springer, New York (2004)

10. Kenmochi, Y., Imiya, A., Ichikawa, A.: Boundary extraction of discrete objects. Computer Vision and Image Understanding 71, 281-293 (1998)

11. Munkres, J.R.: Elements of Algebraic Topology. Addison-Wesley Co. (1984) 
12. Peltier, S., Alayrangues, S., Fuchs, L., Lachaud, J.O.: Computation of homology groups and generators. Computers \& Graphics 30(1), 62-69 (2006)

13. Peltier, S., Ion, A., Haxhimusa, Y., Kropatsch, W.G., Damiand, G.: Computing Homology Group Generators of Images Using Irregular Graph Pyramids. In: Escolano, F., Vento, M. (eds.) GbRPR 2007. LNCS, vol. 4538, pp. 283-294. Springer, Heidelberg (2007)

14. Real, P., González-Díaz, R., Jimenez, M.J., Medrano, B., Molina-Abril, H.: Integral Operators for Computing Homology Generators at Any Dimension. In: RuizShulcloper, J., Kropatsch, W.G. (eds.) CIARP 2008. LNCS, vol. 5197, pp. 356-363. Springer, Heidelberg (2008)

15. Zomorodian, A., Carlsson, G.: Localized Homology. Computational Geometry: Theory \& Applications (to appear)

16. Zomorodian, A.: Topology for Computing. Cambridge University Press, New York (2005) 